Review

\title{
Physical bases of Boron Neutron Capture Therapy, dosimetry, and its mechanisms of action - a critical overview of the litera- ture.
}

\author{
Dominika Skwierawska 1,5, Marcin Balcerzyk ${ }^{2,3, *}$, Jose Antonio Lopez-Valverde ${ }^{2,4}$, Antonio Leal ${ }^{2,4 *}$ \\ 1 Gdańsk University of Technology, Faculties of Applied Physics and Mathematics; Electronics, Telecommu- \\ nications, and Informatics; Chemistry, Gabriela Narutowicza 11/12, 80-233 Gdańsk, Poland \\ 2 Departamento de Fisiología Médica y Biofísica, Facultad de Medicina, Universidad de Sevilla, Spain \\ 3 Unidad Ciclotrón, Centro Nacional de Aceleradores, Universidad de Sevilla-CSIC-Junta de Andalucía, \\ Spain \\ 4 Instituto de Biomedicina de Sevilla (IBiS), Universidad de Sevilla - CSIC - Junta de Andalucía, Spain \\ 5 Current address: MERK Sp. z o.o., Mikołaja Reja 3, 80-404 Gdańsk, Poland \\ * Correspondence: e-mail: mbalcerzyk@us.es, alplaza@us.es
}

\begin{abstract}
The success of boron neutron capture therapy(BNCT) mainly depends on sufficient spatial biodistribution of boron $\left({ }^{10} \mathrm{~B}\right)$ localized around or within the neoplastic cells to produce a high dose gradient between the tumor and healthy tissue. Contrary to what is usual in radiotherapy, BNCT proposes treatment planning directed at the cell instead of the tumor mass. However, it is not yet possible to precisely determine the concentration of ${ }^{10} \mathrm{~B}$ in a specific tissue in real-time using noninvasive methods. Some critical issues still need to be resolved if BNCT is to become valuable, minimally invasive, and efficient cancer treatment. This review article provides an overview of fundamental principles, the recent advances, and future directions of BNCT as cellular targeted cancer therapy. The main emphasis is on topics related to biological dosimetry, methods for assessment of boron concentration, mechanisms of action of BNCT, and its physical bases for clinical implementation.
\end{abstract}

Keywords: boron neutron capture therapy (BNCT); boron imaging; biological dosimetry; radiation therapy

\section{Introduction}

Cancer, a multicellular and multigenic disease, is one of the leading causes of death in the world. It is the first/second cause in 112 of 183 countries and third/fourth in 23 countries according to estimated data from the World Health Organization (WHO) in 2019 [1]. In 2020, 19.3 million new cases and 10 million cancer deaths were registered according to the GLOBOCAN estimates of 36 Cancers in 185 Countries [2]. Given that the yearly incidence continues to increase, the clinical management of cancer remains a significant challenge. Cancer can arise from all organs and different cell types with a multi-factorial etiology. In general, cancerous cells exhibit inherent phenotypical characteristics, known as the hallmarks of cancer. Hanahan and Weinberg [3] originally suggested six alterations in cell physiology that collectively dictate malignant growth: metastasis to different parts of the body, tissue invasion, sustained angiogenesis, evasion of apoptosis (programmed cell death), environmental independence for growth, and unlimited proliferative potential. In a more recent update, they also included deregulated metabolism and immune system evasion as additional hallmarks, as well as two characteristics enabling the acquisition of those hallmarks: genome instability and inflammation [4].

In countries with a high gross domestic product, Radiotherapy (RT) is used in more than $50 \%$ of patients either to treat the disease at a local stage or to control and alleviate the symptoms of irrecoverable cases, depending on the cancer stage [2]. RT aims to deliver 
the optimal dose to the tumor volume while sparing the normal tissues, but these volumes are considered at a macroscopic level in the planning treatment by following an evidencebased population medicine approach. To achieve more efficient personalized treatment, it is desirable to select the cells to be treated, reducing the damage to the healthy ones.

Nowadays, estimations about the global demand of RT by cancer patients indicate that, for $87 \%$ of new cases of breast cancer patients, RT is the treatment of choice according to clinical guidelines and evidence-based medicine, whether applied by itself or in combination with other therapies. The percentage drops to $74 \%$ for head and neck cases, with local control at five years achieved in $34 \%$ of cases and overall survival of $20 \%$ [5]. In other disease locations, RT is usually required as a prophylactic agent after surgery. Nevertheless, in locations like lungs, the radiation beams accuracy allows using RT under ablative conditions when surgery is not applicable. This is the case of Stereotactic Ablative Radiotherapy (SABR), where vascular endothelial injury and immune activation are new radiobiological aspects that must be added to the reoxygenation, repair, and redistribution, for explaining the ablation effect.

These data indicate that the role of RT increased its prominence compared to surgery and chemotherapy in the treatment of localized solid tumors, the most widespread expression of cancer. Among other reasons, these achievements are the result of several innovative therapeutic methods and technology improvements, such as the implementation of devices to shape and adapt the irradiation beam to the tumor volume safeguarding organs at risk, or the successful implementation of advanced imaging procedures used in planning and treatment [6]. The RT goal is to achieve more accurate and efficient dose delivery to organs and tissues [7]. The patients often vary between tumor responses to RT due to differences in tumor type and other specific genetic factors not considered in the treatment planning. Unfortunately, the dose prescription is essentially a population-based approach through the target volume segmentation in the image data of patients usually provided from CT devices.

Boron Neutron Capture Therapy (BNCT) is a high linear energy transfer (LET) radiation therapy used for cancer treatment. Boron can be selectively localized in tumor cells. Thus, BNCT is a promising disease-targeted therapy as neutrons kill preferentially the cells, which are labeled with ${ }^{10} \mathrm{~B}$. A fundamental principle of this method is ${ }^{10} \mathrm{~B}(\mathrm{n}, \alpha){ }^{7 \mathrm{Li}}$ nuclear reaction, which occurs when the stable isotope ${ }^{10} \mathrm{~B}$, delivered preferentially to the tumor cells, is subsequently irradiated with an external epithermal neutron beam to produce an $\alpha$-particle $\left({ }^{4} \mathrm{He}\right)$ and a ${ }^{7} \mathrm{Li}$ nucleus. A schematic representation of this reaction is presented in Figure 1. Released $\alpha$-particles $(\sim 1.47 \mathrm{MeV})$ and ${ }^{7} \mathrm{Li}(\sim 0.84 \mathrm{MeV})$ nuclei have high LET $\sim 175 \mathrm{keV} / \mu \mathrm{m}$ [8-10]. About $94 \%$ of the time, the recoiling ${ }^{7} \mathrm{Li}$ ion is produced in an excited state and emits a low LET $477 \mathrm{keV}$ gamma-ray during deexcitation. In the remaining $6 \%$ events, the ${ }^{7} \mathrm{Li}$ is emitted with no gamma-ray emission in the ground state.

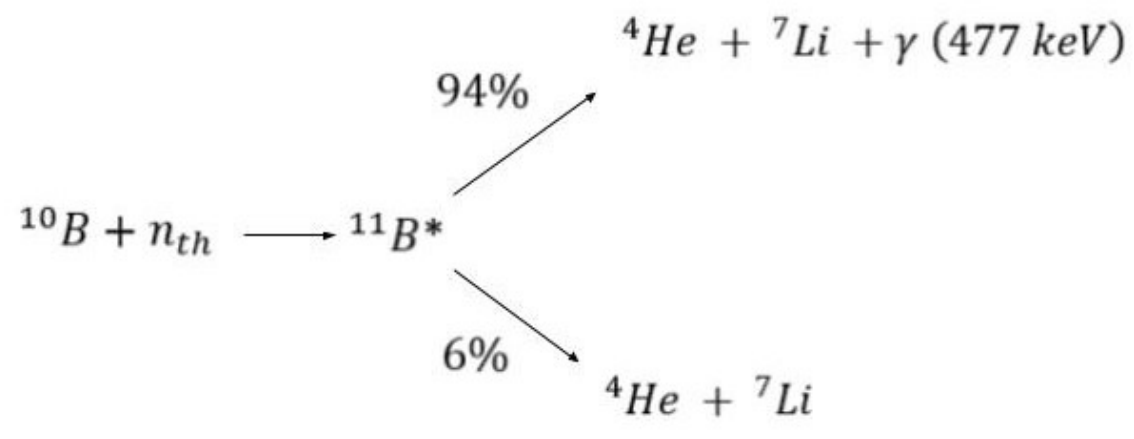

Figure $1 .{ }^{10} \mathrm{~B}(\mathrm{n}, \alpha)^{7} \mathrm{Li}$ reaction.

Unfortunately, after many years of research led by scientists and specialists, they are still struggling with some critical issues of BNCT. Firstly, generating a therapeutic beam with an optimal energy spectrum that can deliver the neutrons to the correct location 
while minimizing the dose delivered to healthy tissue. Secondly, finding non-toxic ${ }^{10} \mathrm{~B}$ delivery agents: boron carrier compound is one of the fundamental aspects of BNCT and should bring significantly more isotope to the cancerous cells than to the healthy tissue or, ideally, only into the cancerous cells. It should also meet requirements like water solubility, chemical stability, and preservation of a constant high concentration during the treatment [11]. As the boron concentration level directly affects the intensity of the boron neutron capture reaction and hence the dose to the tumor and other tissue, it is also essential to image the boron distribution while considering BNCT. Several modalities are widely used to assess boron dose delivered to the residual tumor volume, and they can provide information about the ${ }^{10} \mathrm{~B}$ distribution at the microscopic level $[12,13]$. To improve molecular imaging, several approaches have been proposed [14-17]. Another critical issue is developing treatment planning programs and systems to calculate the dose, predict the particle fluxes and expect the incidence angles on the patients to achieve the adequate relative dose distribution. If the reactor generates the BNCT treatment beams, the treatment planner must determine the dose induced by neutrons and gamma photons. In this case, most of the gamma photons occurring in the beam originating from the reactor core. Fortunately, development research on compact, in-hospital Accelerator-Based Neutron Source, ready for installation in hospital environments, has been ongoing for many years. It allowed more widespread use of the BNCT technique [18]. First, Europe's AcceleratorBased Boron Neutron Capture Therapy Platform designed by Neutron Therapeutics was installed in the Helsinki University Hospital. It is driven by a 2.6 MV electrostatic accelerator operating at $30 \mathrm{~mA}$ proton current on target. Solid lithium with the rotating design was used as a neutron-generating target. Accelerator can distribute over a large area the $78 \mathrm{~kW}(30[m A] \times 2,6[M V]=78[\mathrm{~kW}])$.

There are numerous reasons which define why the cell, after BNCT treatment, may die. They relate mainly to the cell cycle phase in which irradiation occurs, the cell type, the radiation dose, and the oxygen supply. However, what drives cell cancer during switching from a repair program to cell death, and what drives the cancerous cell to choose a specific pathway of death? The specific mechanism of cell death and the mechanism of the repair after BNCT are not sufficiently known. Evaluating early and late markers of the cellular responses after introducing BNCT should be considered in this case. They are crucial for the further development of BNCT.

This review highlights the outlined issues and evaluates them in the future directions and further development of Boron Neutron Capture Therapy as an effective cancer treatment. Later in the article, the possible mechanisms of cell death induced by BNCT are described, and its different types will also be presented. Answer for presented and other questions that will undoubtedly arise in the further development of research on cancer radiotherapy and the progression in solving the issues presented, even in part, will finally lead to continued improvement in BNCT cancer treatment [14,19].

\section{Mechanisms of action of BNCT}

Two types of neutron beams are commonly used in BNCT: thermal beams $(\sim 0.0254$ $\mathrm{eV})$ and epithermal beams $(0.5 \mathrm{eV}$ to $40 \mathrm{keV})$. For clinical purposes, the most useful are epithermal neutrons because, while entering the tissue, they create a radiation field with maximum thermal flux at a depth of $2-3 \mathrm{~cm}$, which after that drops exponentially. In turn, when a thermal beam is entering a tissue, the thermal flux, which is created as a result, falls off exponentially from the surface. For BNCT in the boron-labeled tumor cells, an adequate thermal neutron field must be created. Therefore, a neutron source compliant with the International Atomic Energy Agency (IAEA) standards is required. [20]

Until recently, the value of BNCT was largely restricted, and the number of patients treated using BNCT was very limited because the treatment could be performed only in the nuclear research reactors - the only neutron source at the time. Also, most of the conducted clinical trials were based in facilities at nuclear reactor sources. However, with the improvement of neutron beams generating instruments, BNCT would be able to damage 
tumor cells more effectively while at the same time avoiding affecting normal peripheral cells.

Boron delivery agents are one of the essential aspects of BNCT. ${ }^{10} \mathrm{~B}$ should be retained in the tumor, at least for the duration of neutron irradiation, which can take up to an hour. However, the way to concentrate ${ }^{10} \mathrm{~B}$ at high enough amounts and in cancer cells preferentially is currently the major limitation of the effectiveness of BNCT. Many compounds have been developed until now, but at the moment, only two boron agent's sodium borocaptate $\left(\mathrm{Na}_{2} \mathrm{~B}_{12} \mathrm{H}_{1} \mathrm{SH}\left[\left[{ }^{10} \mathrm{~B}\right] \mathrm{BSH}\right]\right)$ and $\left[{ }^{10} \mathrm{~B}\right] 4$-borono-L-phenylalanine (BPA) - two low molecular weight boron-containing drugs - are both widely used as boron carriers.

$\mathrm{BSH}$ consists of $12{ }^{10} \mathrm{~B}$ atoms, and it is mainly used to treat malignant gliomas. $\mathrm{BSH}$ is not delivered into the normal brain through the intact blood-brain barrier (BBB), and it is difficult to selectively internalize BSH in tumor cells because of its high hydrophilicity. Its concentration in the target relates to the concentration of the agent in the blood and vascularization of the neoplasm [21]. BSH has a passive diffuse accumulation mechanism. In malignant cells in the brain, it accumulates only in the tumor region where the bloodbrain barrier is disrupted [22].

On the other hand, BPA is a derivative of phenylalanine, and it is actively transported into tumor cells, mainly through L-type amino acid transporter 1 (LAT 1) [23]. BPA $\operatorname{logP}$ is negative (-3.65), and this indicates that it will not pass BBB passively because only small molecules with $\log P$ in the range $+1 \ldots+2$ can cross $\mathrm{BBB}$ with passive (diffusive) transport $[24,25]$. Therefore, the only solution for BPA to cross BBB is to sneak it in through some transporter, like L-type amino acid. BPA has been reported to specifically accumulate in tumor cells because of its similar structure to tyrosine [26,27]. The structure of tyrosine and phenylalanine is visualized in Figure 2. As L-type amino acid transporters are involved in some important human diseases and overexpressed in human tumors, it improves targeted delivery into the brain and cancer cells. LAT 1 is also present in the bloodbrain barrier (BBB), blood-retina barrier, cerebral cortex, testis, placenta, and bone marrow. Injection of $\left[{ }^{10} \mathrm{~B}\right] \mathrm{BPA}$ for intravenous administration in BNCT is prepared as the $\left[{ }^{10} \mathrm{~B}\right] \mathrm{BPA}-$ fructose complex [28]. The reason for tagging compounds with positron emitters is to accurately determine the boron distribution and concentration in tumor and surrounding tissue using PET. BPA was approved in Japan as a commercial drug with social security reimbursement, and since May 20th, 2020, it has been available on the market under the name Borofalan $\left({ }^{10} \mathrm{~B}\right)[29]$.<smiles>N[C@@H](Cc1ccccc1)C(=O)O</smiles><smiles>N[C@@H](Cc1ccc(O)cc1)C(=O)O</smiles>

Figure 2. Amino acid chemical structures: phenylalanine (top), tyrosine (bottom)

Three generations of boron compounds can be distinguished: (I) Boric acid and its derivatives used in the first clinical trials. (II) Boron-modified amino acids. This group included boron carriers like BPA, BSH, Na2 ${ }_{12} \mathrm{H}_{11} \mathrm{SH}$, and (L)-4-dihydroxy-borylphenylalane. (III) The third generation of boron agents attracted the attention of scientists over 
the past two decades. They are focused on using biochemical pathways to accumulate boronated analogs in subcellular structures. Those new BNCT agents include boron-containing small molecules, peptides, antibody-based delivery systems, boron compound conjugates, boron delivery nanoparticles (nanomaterial-based delivery systems), and many others currently under evaluation. Targeted boron delivery agents combine boroncontaining agents with tumor-targeting molecules (e.g., nucleosides, porphyrins, peptides, proteins, or antibodies). Boron-delivery nanomaterials can transport various boroncontaining compounds into the tumor cells by taking advantage of nanomaterials' enhanced permeability and retention effects and the active targeting effects mediated by tumor-targeted ligands grafted on the surface of the materials. The following boron-delivery nanomaterials can be distinguished: dendrimers, liposomes, polymeric nanoparticles, boron nitride, carbon nanotubes, mesoporous silica nanoparticles, ferromagnetic and paramagnetic nanoparticles - some of them used for MRI imaging, - gold nanoparticles, and $\mathrm{BPO}_{4}$ nanoparticles. There are many promising routes in drug delivery systems, and there is still a pressing need to develop new boron delivery agents, but without adequate research and clinical trials, it is hard to determine which one is the most feasible [30,31].

\subsection{Physical bases and fundamental dosimetric process of BNCT}

Ionizing radiation has many forms, from alpha, beta, proton, or neutron particles to $X$ or gamma rays and others. In Boron Neutron Capture Therapy, components that contribute to the total absorbed dose rate are due to elastic interaction of incident neutrons with hydrogen, gamma ray's dose emitted by the source, and thermal neutrons captured by hydrogen, nitrogen, and boron [32]. Each of the components has various biological weighting factors. The total biologically absorbed dose (Gy-Eq) is the sum of physical dose components (D) multiplied by the compound biological effectiveness (CBE) or relative biological effectiveness (RBE) of each dose component. RBE is the ratio of the dose absorbed from the reference radiation to the value of the tested radiation dose, producing the same biological effect, whereas CBE stands for values of the biological efficacy for each dose component, depending on what boron carrier was used. CBE differs from RBE with high LET general radiation in that the value varies with the target cells and/or tissue and type of boron compounds used [33]. However, because of the occurrence of events at the cellular and subcellular levels, the different energies and types of radiation involved, the dosimetry and accurate estimation of the RBE, CBE, and therefore biological effectiveness of BNCT is challenging.

Streitmatter et al. [34] presented a multiscale system of dosimetry and radiobiological models that better assess biological effectiveness. It can predict not only the CBE and RBE but also other critical biological metrics for neutron sources, like boron microdistribution and tissue types. The model was tested against results from published experiments in vitro and in vivo, with and without boron, and it showed good agreement between both.

Human tissue also contains certain isotopes that react with neutrons. Due to the values of nuclear cross-sections, most meaningful interactions of neutrons with human tissue involve ${ }^{1} \mathrm{H},{ }^{12} \mathrm{C},{ }^{14} \mathrm{~N}$, and ${ }^{16} \mathrm{O}$ isotopes, which account for $99.2 \%$ of all atoms in the human body [35]. The types of occurring DNA lesions - base damage, crosslinks DNA-protein or DNA-DNA, double-strand breaks (DSB), single-strand break (SSB), sugar-phosphate backbone interruption etc. -, the distribution of these DNA lesions, and the repair pathways depend strongly on the type of radiation used during BNCT, and its LET characteristics $[11,36]$. Consequently, all described components should be considered during the evaluation and calculation of the received doses because they could also be responsible for the adverse effects of BNCT. The most advanced methods of calculating the fluxes and doses in complex geometries with a heterogeneous physical density like the ones in the patient are based on Monte Carlo techniques. Four major absorbed dose components in BNCT can be distinguished:

1. Fast neutron dose: according to ${ }^{1} \mathrm{H}(\mathrm{n}, \mathrm{n}) \mathrm{p}$ reactions, fast and epithermal neutrons cause elastic neutron collisions with hydrogen in tissue (giving recoiling protons and 
gammas). Other energy depositions from fast neutron reactions like ${ }^{12} \mathrm{C}(\mathrm{n}, \alpha)$ are also included.

2. Incident and secondary gamma ray's dose: Primary gamma dose from the beam port and secondary gamma dose by ${ }^{1} \mathrm{H}(\mathrm{n}, \gamma)^{2} \mathrm{H}$.

3. Nitrogen dose: according to ${ }^{14} \mathrm{~N}(\mathrm{n}, \mathrm{p}){ }^{14} \mathrm{C}$ reaction, ${ }^{14} \mathrm{~N}$ element in tissue captures a thermal neutron and, as a result, a $\sim 600 \mathrm{keV}$ proton is emitted. The dose is obtained from locally delivered energy from the recoiling ${ }^{14} \mathrm{C}$ nucleus and the energetic proton.

4. Boron dose: energy deposited by the ${ }^{10} \mathrm{~B}(\mathrm{n}, \alpha)^{7 \mathrm{Li}}$ reaction. ${ }^{10} \mathrm{~B}$ captures a thermal neutron and, as a result, an alpha particle and recoiling ${ }^{7} \mathrm{Li}$ ion are emitted. The dose derived from the reaction products is $\sim 2,31 \mathrm{MeV}$.

The cross-sections for some of those reactions are shown in Figure 3.

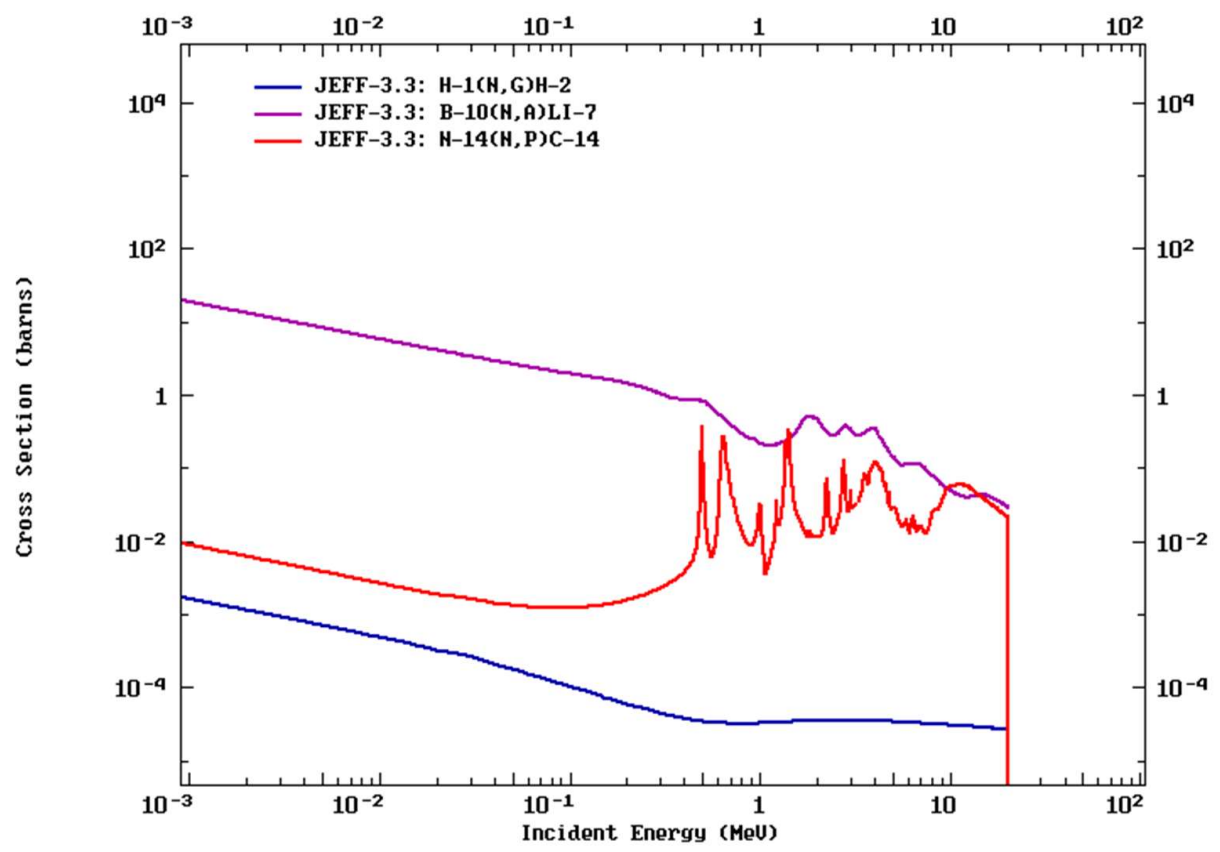

Figure 3. Cross-sections of reactions that take place because of neutron collisions. ${ }^{1} \mathrm{H}(\mathrm{n}, \gamma)^{2} \mathrm{H}$ (blue), ${ }^{10} \mathrm{~B}(\mathrm{n}, \alpha)^{7} \mathrm{Li}$ (magenta), ${ }^{14} \mathrm{~N}(\mathrm{n}, \mathrm{p}){ }^{14} \mathrm{C}$ (red) [37].

Recoil ionization of hydrogen is the leading way by which neutrons with energy $>0.01 \mathrm{MeV}$ are depositing dose. However, the ${ }^{14} \mathrm{~N}(\mathrm{n}, \mathrm{p}){ }^{14} \mathrm{C}$ reaction at neutron energies $<1 \mathrm{eV}$ is responsible for $\sim 80 \%$ of the energy released in tissue [35]. 88.8\% of thermal neutrons are absorbed in the ${ }^{1} \mathrm{H}(\mathrm{n}, \gamma)^{2} \mathrm{H}$ reaction, and $10.6 \%$ of thermal neutrons are absorbed in ${ }^{14} \mathrm{~N}(\mathrm{n}, \mathrm{p}){ }^{14} \mathrm{C}$ reactions. Additionally, in the reactions mentioned above, the ${ }^{14} \mathrm{~N}$ atom also loses an electron. However, proton and electron are not combining instantly as ${ }^{1} \mathrm{H}$. For this, the proton is moving too rapidly through tissue $(\mathrm{Q}=0.58 \mathrm{MeV})$ and will cause further ionization due to the high LET. The emitted proton average residual range in soft tissue (after entering the high-LET Bragg peak phase) is longer than the diameter of a typical cell nucleus but shorter than the diameter of a typical human cell. It is also necessary to estimate the cell-killing potential of the ${ }^{14} \mathrm{~N}(\mathrm{n}, \mathrm{p}){ }^{14} \mathrm{C}$ reaction and consider it since adenosine of ATP, ADP, AMP, DNA and RNA bases and other common molecules such as NADH contain a significant amount of nitrogen. In a tissue exposed to a dose arising from a fast neutron beam, the cells killed by ${ }^{14} \mathrm{~N}(n, p){ }^{14} \mathrm{C}$ reactions compared with those killed by recoil proton and heavy-ion tracks are imperceptible [35]. Additionally, doses from ${ }^{14} \mathrm{C}$ decay compared to background radiation and statutory limits are insignificantly smaller. The fraction of respiratory phosphates molecules - i.e., AMP, ADP, ATP, NADH, etc. that undergo the ${ }^{14} \mathrm{~N}(\mathrm{n}, \mathrm{p}){ }^{14} \mathrm{C}$ reaction is negligible at therapeutic neutron doses. 
Determination of the dose resulting from the reaction ${ }^{14} \mathrm{~N}(n, p){ }^{14} \mathrm{C}$ is necessary for situations where people may be exposed to prolonged exposure to significant thermal neutron fluxes [35].

As a result, the dosimetry of BNCT requires an in-depth analysis of various components of the radiation field. To predict a biological effect, the dose arising from each of these four components must first be multiplied by an appropriate biological weighting factor to account for differences in relative biological effectiveness and, in the end, combined [20]. Accepted values of the biological weighting factors are 1.3 for boron dose in normal tissues, 3.8 for boron dose in tumor, 3.2 for thermal and fast neutron dose, 1 for gamma dose.

\subsection{Mechanisms of cell death}

In BNCT, the cells are mainly killed when the alpha particle or ${ }^{7} \mathrm{Li}$ ion causes various DNA lesions leading to genome instability. During BNCT treatment, clustered DNA damages or locally multiple damage sites may occur. Besides DNA, cellular macromolecules can also be damaged, which can cause modulation of their functions [39]. All of this happens when a heavy particle passes through the cell nucleus. The reaction products have a concise range $\left(\alpha<10 \mu \mathrm{m}\right.$ and $\left.{ }^{7} \mathrm{Li}<5 \mu \mathrm{m}\right)$ [40], so kinetic energy is delivered to the target cell, whose diameter is usually $\sim 10 \mu \mathrm{m}$. Therefore, it does not affect surrounding healthy cells. Intracellular localization of boron is critical because the normal healthy tissue can be spared from the nuclear reactions if they have not taken up ${ }^{10} \mathrm{~B}$. Unfortunately, with the currently available boron carrier compounds, some of ${ }^{10} \mathrm{~B}$ also accumulate in healthy cells. The development of boron carriers is still very active today [41].

During and after irradiation, the damage occurs at the cellular level. The damage can be divided into two groups - direct and indirect action mechanisms. In the first one, the radiation affects the DNA directly, which causes the ionization of the atoms within the DNA molecule. However, ionization caused by radiation must take place within a few nanometers of the DNA molecule for this action to take place. In the second scenario of indirect action, the radiation interacts with other target molecules or atoms, which it encounters - usually water [42]. As a result, highly reactive species (like $\mathrm{HO} \cdot$ and $\mathrm{H} \cdot$ ) which can diffuse some distances in the cell are produced. These free radicals are formed in irradiated body tissue and blood cells.

DNA damage increases together with LET of radiation [43], and the higher the LET, the higher the relative biological effectiveness (RBE). The radiation field generated during BNCT consists of components with different LET characteristics that act independently. Low-LET radiation ionizes sparsely, while high-LET radiation causes denser ionization along the track and can lead to more complex DNA damages. DNA damage after highLET radiation remains unrepaired for a long time, leading to genome instability or death of the cell [44]. The density of radiation affects the presence and quality of the radiationinduced DSB.

Like many cancer treatments, radiation therapy achieves its therapeutic effect by causing a reaction of different types of cell death: apoptosis, mitotic cell death or mitotic catastrophe, necrosis, autophagy, and others. Apoptosis or mitotic cell death are the most common types. Apoptosis induced by radiation is a progressive and degradative process. Extrinsic and intrinsic apoptosis can be distinguished. Intrinsic apoptosis is a type of regulated cell death (RCD) initiated by perturbations of the intracellular microenvironment and demarcated by mitochondrial outer membrane permeabilization. Mitotic cell death is one specific variant of RCD driven by mitotic catastrophe, an oncosuppressive mechanism to control mitosis-incompetent cells [45]. Wang et al. [46] confirmed that in glioma cells, BNCT-induced apoptosis was mediated by the Bcl-2/Bax pathway.

Another important goal of radiation therapy is to deprive cancer cells of their potential to divide and multiply indefinitely [7]. The primary and presumed cell target of the ionizing radiation is DNA itself. However, damages or mutations of different cellular macromolecules cannot be eliminated entirely, and as a result, their functions could be 
modulated, and other subsequent biological changes can be observed after cancer treatment [39].

The DNA-DSB repair process is complex and depends on many factors, including cell cycle phase and checkpoints, DSB-inducing agents, ncRNAs, and various gene mutations characterized by different cancer cell lines. Several attempts to investigate specific cellular DNA damage response induced by mixed neutron-gamma field has been taken over the last years [44,47-49]. Despite that, this phenomenon is not fully understood and determined.

Rodriguez et al. [50] have attempted in vitro studies of DNA damage and repair mechanisms induced by BNCT. The human thyroid follicular cancer cell line was used for the research. The evaluation of DNA damage was made by detecting H2AX histone phosphorylation foci ( $\mathrm{YH} 2 \mathrm{AX}$ foci). Two mediating pathways repair of the DBS have been identified in mammalian cells, HRR and NHEJ. After follicular carcinoma cells analysis, repair pathways were observed with an increase of Rad51 and Rad54 mRNA expression (at 4 and $6 \mathrm{~h}$ after the irradiation) showing the expression of enzymes belonging mainly to the HRR pathway specify a different DNA damage pattern and showed activation of both repair pathways. However, what exactly determines the activation of HRR or NHEJ is not yet completely clear.

To increase anti-cancer biological activity during BNCT therapy, Ikuhiko Nakase et al. performed an in vitro BNCT assay [51]. The study also assessed the cell death pathways to understand cell-killing activity occurring after thermal neutron irradiation. They synthesized and demonstrated organelle-targeted cell-penetrating peptide (CPP)-conjugated boron compounds. CPPs help to control intracellular localization, cell membrane penetration and further enhance cellular uptake of the boron compound. This controlled delivery affects the cell death types and efficacy of the cancer cell killing activity. Treatment of DBRLA ((BODIPY) - labeled dodecaborates conjugated to the RLA peptide) showed a higher reduction of ATP content than other tested peptides. ATP depletion enhances necrosis, which consequently might induce necrosis in BNCT. This could be one of the significant factors of the cell-killing activity- detailed mechanisms should be further studied.

\subsection{BNCT biological dosimetry}

As it was noted previously, the BNCT effect relies heavily on a biological component. Thus, it is crucial to assess the increased radiosensitization effect promoted, besides the physical dose enhancement. This is a hard task that depends specifically on in vivo or in vitro studies, involving methodologies such as proliferation tests, clonogenic tests, or DNA damage evaluation.

Sung et al. [52] performed clonogenic tests, evaluating the survival in terms of the proliferative capacity of irradiated cells, and obtained a dose-dependent suppression of cell survival when they were treated with BPA under BNCT irradiation schemes. This effect showed up to 10 times less survival when boron was present during a $\sim 3$ Gy irradiation. Furthermore, they also analyzed the mitochondrial metabolic activity of irradiated cells with the 3-(4,5-dimethylthiazol-2-yl)-2,5-diphenyl tetrazolium bromide (MTT) assay. The results showed a significant decrease of metabolic activity in different cells irradiated with BPA relative to cells irradiated without BPA, ranging from $\sim 20 \%$ up to $~ 80 \%$ at 3 days after irradiation, depending on the cell line assessed. This result suggested a decrease in proliferative capacity after BNCT. In addition, they also pointed out a cell cycle arrest in G2/M checkpoints and an increase of apoptotic cells after BNCT vs neutron irradiation, using flow cytometry assays. The increase of apoptotic cells and cell cycle arrest in G2/M were confirmed in terms of increased expression of caspase- 9 and cytochrome $\mathrm{c}$ and decreased expression of cyclin B1 and CDK1, respectively, using western blots. These results are consistent with other studies' reports [53,54]. Moreover, newer studies even proposed mathematical models fitting data from experiments studying the same biological-effectiveness-related cellular parameters [55]. 
In any case, the radiosensitization effect could be observed at a more precise level in terms of DNA damage. Thus, studies evaluating the presence of DSB-repair markers such as $\mathrm{YH} 2 \mathrm{AX}$ foci have provided further insights into the matter. This is the case of the study carried out by Rodriguez et al. [50], which determined that the number of localized lesions was lower when comparing gamma-ray radiation with neutron or BNCT radiation, but the damage caused by BNCT was densely concentrated in clusters, which correlates with the expected more complex damage caused by high LET radiation. Moreover, these large foci lesions were persistent when observed for longer timeframes, describing firm or irreparable long-term damage [56,57]. Thus, despite an initial lower $\mathrm{YH} 2 \mathrm{AX}$ foci count, the BNCT DNA damage profile involves more complex and irreparable damage patterns that would mean a higher radiobiological effect. Besides, it is well known that dose rate plays a crucial role in radiosensitization [58]. Hence, the long-term effect of the appearance of such discrete events of a large deposition of dose prompted by BNCT remains to be determined. These events differ heavily from the more continuous events that occur in conventional $y$ irradiation, depositing less dose each one.

At the same time, BNCT treatment has been shown to alter the cellular oxidative stress levels, both because of BNCT itself and because of the tumor-targeting boron carriers $[59,60]$. The effects of these oxidative stress changes in biological effective dose and radiosensitization need to be further studied.

\section{Methods for assessment of boron concentration in residual tumor volume and healthy tissue}

BNCT agents deliver boron atoms precisely to tumor cells, maintaining the appropriate concentration higher in tumor than in normal tissue. The effectiveness of the therapy depends on where the drug was in the population of neoplastic cells and within the tumor cells. Intranuclear localization of boron increases the chances of killing cells by damage to DNA. The lack of a method for a quantitative imaging evaluation of the boron concentration was always one of the issues that nuclear doctors faced while using neutron irradiation. Thus, methods for assessing the three-dimensional distribution of boron drugs, boron dose, and all complex radiation compositions delivered to the residual tumor volume and healthy tissue are one of the most critical issues of Boron Neutron Capture therapy. Chemical imaging of cellular and sub-cellular levels is necessary to support clinical efficacy, dosimetry studies, and generally novel drug delivery research in BNCT. To solve this problem and achieve selective tumor accumulation and reduced toxicity, several approaches like coating, functionalizing, and many others have also been applied. Labeling by different fluorophores or molecules with fluorescence properties was also researched and developed for better imaging $[15,16]$.

The boron concentration level directly affects the boron neutron capture reaction intensity and the dose to the tumor and other tissue. So, it is essential to image the boron distribution while considering BNCT. Therefore, it is necessary to develop and assess alternative methods to predict blood boron levels between measurements and during irradiation. Substantial improvement of BNCT will be achieved when boron concentration is measured in situ. Additionally, it should also be considered that the uptake of the boroncarrying molecules in target cells is heterogeneous. It depends on factors like tumor cellularity (i.e., the number of tumor cells arranged in clusters) [61], cell cycle phase, and others) [62]. It is one of the crucial factors, often marked as a drawback in BNCT, because it causes ambiguity in the calculated dose distributions. For effective BNCT treatment, ${ }^{10} \mathrm{~B}$ concentration ratio in the tumor and its concentration in normal tissues ( $\mathrm{T} / \mathrm{N}$ ratio) should be 3:1 or more, and the ${ }^{10} \mathrm{~B}$ concentration in the target should be at least $\sim 15-30 \mu \mathrm{g} \mathrm{g}^{-1}$ or $\sim 10^{9}$ atoms per cell to execute lethal tumor cell damage [63]. Summarizing, to avoid unfavorable effects, the concentration of ${ }^{10} \mathrm{~B}$ in tumor cells and normal tissues must be known. 


\subsection{Positron emission tomography and magnetic resonance imaging}

Clinically applicable imaging modalities are positron emission tomography (PET) [64] and magnetic resonance imaging (MRI) techniques ( ${ }^{1} \mathrm{H}$ in BPA) [21,65]. Positron emission tomography has many abilities, such as (I) quantifying biochemical processes, (II) reconstructing the distribution of a boron carrier (this information can be later used in treatment planning), (III) locating and determining the extent of metastasis in the body, (IV) predicting the optimal time of neutron exposure to BNCT, (V) controlling the therapeutic effects, and (VI) assessing whether the patient is suitable for BNCT. The suitability of positron emission tomography for the establishment of boron concentration in healthy tissues and tumors and the needs of treatment planning have been examined in many studies $[62,66,67]$. However, PET imaging with the current technology can mainly measure the boron distribution before the treatment. As a result, therapeutic dose distribution calculated with PET can disagree with the actual delivered dose. The most common radiolabelled derivative of BPA used for estimating BPA concentration in vivo through PET is $\left[{ }^{18} \mathrm{~F}\right] \mathrm{FBPA}$. Figure 4 shows the structure of both BPA and $\left[{ }^{18} \mathrm{~F}\right] \mathrm{FBPA}$. Scientists are still conducting studies to detect a compound with a greater potential for noninvasively quantitating local boron concentration via PET imaging, e.g., theranostic agent - metabolically stable boron-derived tyrosine [68].<smiles>N[C@@H](Cc1ccc(B(O)O)cc1)C(=O)O</smiles><smiles>N[C@@H](Cc1ccc(B(O)O)cc1F)C(=O)O</smiles>

Figure 4. Structure of BPA (left) and $\left[{ }^{18} \mathrm{~F}\right] \mathrm{FBPA}$ (right).

MRI also performs well as a modality for indirect quantification of the in vivo boron distribution at the target site, during and before neutron irradiation [69]. It can provide functional and morphological information without using radiation, which makes it safer. For this purpose, to obtain high-contrast images, it is necessary to introduce into the body non-toxic ${ }^{10} \mathrm{~B}$ molecular compounds tagged with a paramagnetic ion, like gadolinium, which will work as an MRI reporter during mapping the boron distribution [70-72].

In the paper [73], Balcerzyk et al. explored the possibility of PET measurement of boron concentration if the compound contains $R-\mathrm{BF}_{3}$ moiety labeling it with ${ }^{18} \mathrm{~F}$. This method was applied to $\left[{ }^{18} \mathrm{~F}\right] \mathrm{NaBF}_{4}$ used in thyroid cancer preclinical study.

However, measurement of the net content of ${ }^{10} \mathrm{~B}$ atoms - bound and free pools of boron - and factors affecting the net in individual tumor cells are not described broadly in literature and remains challenging since both PET and MRI modalities do not offer sufficient spatial resolution to quantify boron atoms in single cells [74].

\subsection{Mass spectrometry imaging}

Mass spectrometry imaging (MSI) is a powerful tool capable of imaging and profiling various molecules with high sensitivity - subcellular structures and individual cells without labeling in a single experiment, e.g., intracellular localization of pharmaceuticals. However, the disadvantage is that using MSI absolute quantification is usually not possible as opposed to secondary ion mass spectrometry (SIMS), due to the diversity of factors that affect the intensities of ion signals recorded within the region of interest. 
SIMS operates in the MSI mode, and it can routinely achieve spatial resolutions at the submicron level. Therefore, it is a powerful tool often used in micro-bioanalytical investigations and drug distribution studies [75]. Due to this dynamic, SIMS was used quantitative mapping of boron directly at subcellular resolutions, which allowed for a successful evaluation of the effectiveness of various BNCT pharmaceuticals and to compare boron concentration in subcellular regions [76].

Two directions of studies focused on using SIMS in BNCT can be distinguished: (I) microprobe methods combined with laser post-ionization techniques, and (II) use of the ion microscope technique applying a high-current, primary beam $\mathrm{O}_{2}{ }^{+}$and afterward with use of position-sensitive detector detecting positive secondary ions [63].

Chandra et al. successfully performed many SIMS-based investigations and quantitative evaluations over boron neutron capture therapy drugs. The assessment of free or loosely bound boron pools was done in the cytoplasm and the nucleus of cryogenically prepared cultured human glioblastoma multiforme cells exposed to BPA. Both evaluated boron agents delivered $\sim 70 \%$ of the pool of boron in the bound and mobile form to the nucleus and cytoplasm $[77,78]$.

Aldossari et al. [79] in their study also conducted an application study for the localization and quantification of therapeutic levels of the BNCT agent L-para-(dihydroxyboryl)-phenylalanine (BPA) in a primary cell with the use of a high-resolution dynamic SIMS instrument. Cell cultures were obtained from patients (humans) who suffered from glioblastoma multiforme tumors.

\subsection{Single-photon emission computed tomography and prompt gamma photons detectors}

Much feasibility analysis of a Single-photon emission computed tomography (SPECT) for quantifying the boron dose has been carried out over the years. However, some of them, like the BNCT-SPECT method, would provide only dosimetric data, like an absolute number of BNCT reactions occurring within the measured region. [80,81]. Later, modification of BNCT SPECT [82], able to extract information allowing to determine the boron concentration in real-time, was proposed. It is based on the number of neutrons that pass the patient, measured by taking advantage of the cadmium neutron capture reaction ${ }^{113} \mathrm{Cd}(\mathrm{n}, \gamma)$ occurring in the detector.

Imaging the prompt gamma (PG) photons resulting from the ${ }^{10} \mathrm{~B}(\mathrm{n}, \alpha)^{7} \mathrm{Li}$ reaction is another possible method for detecting boron determination and concentration. It is a similar approach as applied in SPECT. Many different devices like detectors (semiconductor detectors: CZT [83,84], CdTe[85,86]; scintillator detectors [87], and others) have been proposed in the course of numerous researches to promote the clinical translation of this method. Feng et al. [40] proposed a dual prompt gamma detection method that could enable an accurate three-dimensional determination and reconstruction of boron concentration in vivo and dose distribution in the region of interest (ROI) during BNCT. This method is based on the relationship between ${ }^{10} \mathrm{~B}(\mathrm{n}, \alpha)^{7} \mathrm{Li}$ and ${ }^{1} \mathrm{H}(\mathrm{n}, \gamma)^{2} \mathrm{H}$ reactions. However, there are still many technical challenges to solve before implementing this method in clinical applications. 3.4. Other molecular imaging tools in BNCT.

$\mathrm{Hu}$ et al. evaluated Particle and Heavy Ion Transport code System (PHITS) for microdosimetry in BNCT. It can help in the evaluation of dose in radiobiological experiments. Additionally, it can consider intra- and intercellular heterogeneity in the distribution of ${ }^{10} \mathrm{~B}$. Therefore, it was proposed as a model that can estimate the biological effectiveness of newly developed ${ }^{10} \mathrm{~B}$ compounds for BNCT, which would be advantageous in future drug discovery research. The study resulted in a general conclusion that PHITS can be applied in the evaluation of dose rates of absorbed gamma rays and thermal neutron fluxes inside of a tumor imitating medium [88,89].

Worth noting is that boron measurements at the subcellular level in the cytoplasm and nuclei samples collected after fractionation of tumor cells cannot also be made with high confidence by bulk methods of boron determination which are vital to BNCT. Free and loosely bound boron pools would be lost (more likely) from their native subcellular 
locations, e.g., during the liquid centrifugation or in other steps of fractionation. Bulk techniques are not able also to determine the enhanced accumulation of ${ }^{10} \mathrm{~B}$ within the cell nucleus [78].

\section{Conclusions}

Boron Neutron Capture Therapy incorporates the targeting principles of chemotherapy and the anatomical localization principles of conventional radiotherapy. Since some types of cancer, like glioblastoma (GBM), remain exceptionally resistant to all current forms of therapy such as chemotherapy, surgery, radiotherapy, and immunotherapy, Boron Neutron Capture Therapy is a promising option for those tumor types. However, some critical issues need to be resolved if BNCT is to become a better and valuable cancer treatment. Since controlled intracellular targeting has a great significance in inducing the cell-killing activity of BNCT due to specific cell death pathways, such targeting should be further assessed together with performing adequate research and clinical trials to determine the most profitable and promising routes in drug delivery systems. Activation of DNA response, like damage and repair mechanisms of complex Double-Strand DNA Break activated by mixed neutron-gamma beam, have been poorly studied, therefore it is not fully determined. Further understanding of how cells preferentially select specific DNA damage responses generated by high-LET and mixed radiation and detailed mechanisms of enhanced necrosis due to depletion of ADP may lead to improved therapeutic efficiency in BNCT. Individual tumor cell quantification of bound and free pools (net cellular content) of ${ }^{10} \mathrm{~B}$ needs to be further addressed since it remains challenging (due to insufficient spatial resolution) under clinically applicable techniques. Studies designed to test and improve boron detection methods could lead to the reduction of detection limits and identify accumulation regions in tumor cells and normal tissues more precisely. Further research on mechanisms for detecting the distribution of prompt gamma rays arising during BNCT could also be profitable. However, the ideal dosing paradigm for BNCT, real-time measurement of distributions of reactions like ${ }^{10} \mathrm{~B}(\mathrm{n}, \alpha){ }^{7} \mathrm{Li}$ and ${ }^{14} \mathrm{~N}(\mathrm{n}, \mathrm{p}){ }^{14} \mathrm{C}$, and quantitative mapping of boron concentration in the body have yet to be determined.

Author Contributions: D.S. wrote section 1, section 2.1, 2.2 and sections 3.1, 3.2, 3.3, 4. J.A.L.V. wrote section 2.3, contributed to the writing of section 1, and commented and reviewed other sections. All authors have contributed, read, and agreed to the published version of the manuscript. A.L.P. leads the scientific project where this work is supported. A.L.P and M.B. were involved in study design, the style of the manuscript and critically reviewed the text by contributing expertise and guidance.

Funding: This research was funded by the Spanish Ministry of Science, Innovation and Universities, project reference RTI2018-098656-B-I00; The APC was also funded by this research project; D.S. received funding from ERASMUS PLUS - European Commission program for 2014 - 2020, project number 2019-1-PL01-KA103-062953; J.A.L.V. received funding from the FPU call from the Spanish Ministry of Science, Innovation, and Universities, reference number FPU19/02950.

Institutional Review Board Statement: Not applicable.

Informed Consent Statement: Not applicable.

Acknowledgments: Not applicable.

Conflicts of Interest: The authors declare no conflict of interest. 


\section{References}

1. Geneva, W.H.O. Global Health Estimates 2020: Deaths by Cause, Age, Sex, by Country and by Region, 2000-2019. . 2020.

2. Hyuna Sung; Jacques Ferlay; Rebecca L Siegel; Mathieu Laversanne; Isabelle Soerjomataram; Ahmedin Jemal; Freddie Bray BSc Global Cancer Statistics 2020: GLOBOCAN Estimates of Incidence and Mortality Worldwide for 36 Cancers in 185 Countries I Enhanced Reader. 04 February 2021.

3. Hanahan, D.; Weinberg, R.A. The Hallmarks of Cancer. Cell 2000, 100, doi:10.1016/S00928674(00)81683-9.

4. D, H.; RA, W. Hallmarks of Cancer: The next Generation. Cell 2011, 144, 646-674, doi:10.1016/J.CELL.2011.02.013.

5. Atun, R.; Jaffray, D.A.; Barton, M.B.; Bray, F.; Baumann, M.; Vikram, B.; Hanna, T.P.; Knaul, F.M.; Lievens, Y.; Lui, T.Y.M.; et al. Expanding Global Access to Radiotherapy. The Lancet Oncology 2015, 16, doi:10.1016/S1470-2045(15)00222-3.

6. Baskar, R.; Lee, K.A.; Yeo, R.; Yeoh, K.-W. Cancer and Radiation Therapy: Current Advances and Future Directions. International Journal of Medical Sciences 2012, 9, doi:10.7150/ijms.3635.

7. Chen, Y.; Yang, J.; Fu, S.; Wu, J. Gold Nanoparticles as Radiosensitizers in Cancer Radiotherapy. International Journal of Nanomedicine 2020, doi:10.2147/IJN.S272902.

8. Farhood, B.; Samadian, H.; Ghorbani, M.; Zakariaee, S.S.; Knaup, C. Physical, Dosimetric and Clinical Aspects and Delivery Systems in Neutron Capture Therapy. Reports of Practical Oncology \& Radiotherapy 2018, 23, doi:10.1016/j.rpor.2018.07.002.

9. Nedunchezhian, K.; Aswath, N.; Thiruppathy, M.; Thirugnanamurthy, S. Boron Neutron Capture Therapy - a Literature Review. Journal of Clinical and Diagnostic Research 2016, 10, ZE01-ZE04.

10. Macías, M.; Fernández, B.; Praena, J. New Data for the Definition of Neutron Beams for Boron Neutron Capture Therapy. 2020, doi:10.1016/j.radphyschem.2021.109474.

11. Dymova, M.A.; Taskaev, S.Y.; Richter, V.A.; Kuligina, E.V. Boron Neutron Capture Therapy: Current Status and Future Perspectives. Cancer Communications 2020, 40, doi:10.1002/cac2.12089.

12. Espain, M.S.; Dattoli Viegas, A.M.; Trivillin, V.A.; saint Martin, G.; Thorp, S.I.; Curotto, P.; Pozzi, E.C.C.; González, S.J.; Portu, A.M. Neutron Autoradiography to Study the Microdistribution of Boron in the Lung. Applied Radiation and Isotopes 2020, 165, doi:10.1016/j.apradiso.2020.109331.

13. Wittig, A.; Michel, J.; Moss, R.L.; Stecher-Rasmussen, F.; Arlinghaus, H.F.; Bendel, P.; Mauri, P.L.; Altieri, S.; Hilger, R.; Salvadori, P.A.; et al. Boron Analysis and Boron Imaging in Biological Materials for Boron Neutron Capture Therapy (BNCT). Critical Reviews in Oncology/Hematology 2008, 68, doi:10.1016/j.critrevonc.2008.03.004.

14. Dymova, M.A.; Taskaev, S.Y.; Richter, V.A.; Kuligina, E.V. Boron Neutron Capture Therapy: Current Status and Future Perspectives. Cancer Communications 2020, 40, doi:10.1002/cac2.12089. 
15. Qi, P.; Chen, Q.; Tu, D.; Yao, S.; Zhang, Y.; Wang, J.; Xie, C.; Pan, C.; Peng, H. The Potential Role of Borophene as a Radiosensitizer in Boron Neutron Capture Therapy (BNCT) and Particle Therapy (PT). Biomaterials Science 2020, 8, doi:10.1039/D0BM00318B.

16. Wang, D.; Meng, Y.; Wang, X.; Xia, G.; Zhang, Q. The Endocytic Mechanism and Cytotoxicity of Boron-Containing Vesicles. Chemical and Pharmaceutical Bulletin 2020, 68, doi:10.1248/cpb.c19-00971.

17. Cheheltani, R.; Ezzibdeh, R.M.; Chhour, P.; Pulaparthi, K.; Kim, J.; Jurcova, M.; Hsu, J.C.; Blundell, C.; Litt, H.I.; Ferrari, V.A.; et al. Tunable, Biodegradable Gold Nanoparticles as Contrast Agents for Computed Tomography and Photoacoustic Imaging. Biomaterials 2016, 102, doi:10.1016/j.biomaterials.2016.06.015.

18. Kreiner, A.J.; Bergueiro, J.; Cartelli, D.; Baldo, M.; Castell, W.; Asoia, J.G.; Padulo, J.; Suárez Sandín, J.C.; Igarzabal, M.; Erhardt, J.; et al. Present Status of Accelerator-Based BNCT. Reports of Practical Oncology and Radiotherapy 2016, 21, 95-101, doi:10.1016/j.rpor.2014.11.004.

19. Baskar, R.; Lee, K.A.; Yeo, R.; Yeoh, K.-W. Cancer and Radiation Therapy: Current Advances and Future Directions. International Journal of Medical Sciences 2012, 9, doi:10.7150/ijms.3635.

20. IAEA-TECDOC-1223 Current Status of Neutron Capture Therapy. Iaea, 2001 (8) 2001, doi:10.1111/j.1558-5646.2008.00544.x.

21. Miyatake, S.-I.; Kawabata, S.; Hiramatsu, R.; Kuroiwa, T.; Suzuki, M.; Ono, K. Boron Neutron Capture Therapy of Malignant Gliomas. In; 2018.

22. BNCT Chemistry | National Centre for Nuclear Research Available online: https://www.ncbj.gov.pl/en/bnct/chemistry (accessed on 3 September 2021).

23. Puris, E.; Gynther, M.; Auriola, S.; Huttunen, K.M. L-Type Amino Acid Transporter 1 as a Target for Drug Delivery. Pharmaceutical Research 2020, 37, doi:10.1007/s11095-020-02826-8.

24. Cheheltani, R.; Ezzibdeh, R.M.; Chhour, P.; Pulaparthi, K.; Kim, J.; Jurcova, M.; Hsu, J.C.; Blundell, C.; Litt, H.I.; Ferrari, V.A.; et al. Tunable, Biodegradable Gold Nanoparticles as Contrast Agents for Computed Tomography and Photoacoustic Imaging. Biomaterials 2016, 102, doi:10.1016/j.biomaterials.2016.06.015.

25. ChemSpider $\mid$ Search and Share Chemistry Available online: https://www.chemspider.com/ (accessed on 3 September 2021).

26. Baskar, R.; Lee, K.A.; Yeo, R.; Yeoh, K.-W. Cancer and Radiation Therapy: Current Advances and Future Directions. International Journal of Medical Sciences 2012, 9, doi:10.7150/ijms.3635.

27. Suzuki, M. Boron Neutron Capture Therapy (BNCT): A Unique Role in Radiotherapy with a View to Entering the Accelerator-Based BNCT Era. International Journal of Clinical Oncology 2020, 25, doi:10.1007/s10147-019-01480-4.

28. Suzuki, M. Boron Neutron Capture Therapy (BNCT): A Unique Role in Radiotherapy with a View to Entering the Accelerator-Based BNCT Era. International Journal of Clinical Oncology 2020, 25.

29. Borofalan (10B) - Stella Pharma - AdisInsight Available online: https://adisinsight.springer.com/drugs/800045201 (accessed on 16 September 2021). 
30. Barth, R.F.; Mi, P.; Yang, W. Boron Delivery Agents for Neutron Capture Therapy of Cancer. Cancer Communications 2018, 38, doi:10.1186/s40880-018-0299-7.

31. Sauerwein, W.A.G.; Sancey, L.; Hey-Hawkins, E.; Kellert, M.; Panza, L.; Imperio, D.; Balcerzyk, M.; Rizzo, G.; Scalco, E.; Herrmann, K.; et al. Theranostics in Boron Neutron Capture Therapy. Life 2021, 11, doi:10.3390/life11040330.

32. Syamputra, D.N.I.; Sardjono, Y.; Mahmudah, R.S.N. Dose Analysis of BNCT Treatment Method for Rhabdomyosarcoma in the Head and Neck Regions Based on PHITS Code. ASEAN Journal on Science and Technology for Development 2020, 35, doi:10.29037/ajstd.521.

33. Ono, K.; Tanaka, H.; Suzuki, M. Reevaluation of CBE Value of BPA for Hepatocytes. Applied Radiation and Isotopes 2020, 161, doi:10.1016/j.apradiso.2020.109159.

34. Streitmatter, S.W.; Stewart, R.D.; Moffitt, G.; Jevremovic, T. Mechanistic Modeling of the Relative Biological Effectiveness of Boron Neutron Capture Therapy. Cells 2020, 9, doi:10.3390/cells9102302.

35. Swanepoel, M.W. The Role of the $14 \mathrm{~N}(\mathrm{n}, \mathrm{p}) 14 \mathrm{C}$ Reaction in Neutron Irradiation of Soft Tissues. In Proceedings of the Radiation Measurements; 2010; Vol. 45.

36. Freneau, A.; dos Santos, M.; Voisin, P.; Tang, N.; Bueno Vizcarra, M.; Villagrasa, C.; Roy, L.; Vaurijoux, A.; Gruel, G. Relation between DNA Double-Strand Breaks and Energy Spectra of Secondary Electrons Produced by Different X-Ray Energies. International Journal of Radiation Biology 2018, 94, doi:10.1080/09553002.2018.1518612.

37. Evaluated Nuclear Data File (ENDF) Available online: https://www.nndc.bnl.gov/exfor/endf00.jsp (accessed on 13 September 2021).

38. Technology - Neutron Therapeutics Available online: https://www.neutrontherapeutics.com/technology/ (accessed on 17 September 2021).

39. Desouky, O.; Ding, N.; Zhou, G. Targeted and Non-Targeted Effects of Ionizing Radiation. Journal of Radiation Research and Applied Sciences 2015, 8, doi:10.1016/j.jrras.2015.03.003.

40. Tian, F.; Geng, C.-R.; Tang, X.-B.; Shu, D.-Y.; Ye, H.-F.; Bortolussi, S.; Gong, C.-H. Analysis of Influencing Factors on the Method for Determining Boron Concentration and Dose through Dual Prompt Gamma Detection. Nuclear Science and Techniques 2021, 32, doi:10.1007/s41365-021-00873-3.

41. Hu, K.; Yang, Z.; Zhang, L.; Xie, L.; Wang, L.; Xu, H.; Josephson, L.; Liang, S.H.; Zhang, M.-R. Boron Agents for Neutron Capture Therapy. Coordination Chemistry Reviews 2020, 405, doi:10.1016/j.ccr.2019.213139.

42. Elgazzar Abdelhamid H. and Kazem, N. Biological Effects of Ionizing Radiation. In The Pathophysiologic Basis of Nuclear Medicine; Elgazzar, A.H., Ed.; Springer Berlin Heidelberg: Berlin, Heidelberg, 2006; pp. 540-548 ISBN 978-3-540-47953-6.

43. Fujita, Y.; Kato, I.; Iwai, S.; Ono, K.; Suzuki, M.; Sakurai, Y.; Ohnishi, K.; Ohnishi, T.; Yura, Y. Role of P53 Mutation in the Effect of Boron Neutron Capture Therapy on Oral Squamous Cell Carcinoma. Radiation Oncology 2009, 4, doi:10.1186/1748-717X-4-63.

44. Maliszewska-Olejniczak, K.; Kaniowski, D.; Araszkiewicz, M.; Tymińska, K.; Korgul, A. Molecular Mechanisms of Specific Cellular DNA Damage Response and Repair Induced by the Mixed Radiation Field During Boron Neutron Capture Therapy. Frontiers in Oncology 2021, 11, doi:10.3389/fonc.2021.676575. 
45. Galluzzi, L.; Vitale, I.; Aaronson, S.A.; Abrams, J.M.; Adam, D.; Agostinis, P.; Alnemri, E.S.; Altucci, L.; Amelio, I.; Andrews, D.W.; et al. Molecular Mechanisms of Cell Death: Recommendations of the Nomenclature Committee on Cell Death 2018. Cell Death $\mathcal{E}$ Differentiation 2018, 25, doi:10.1038/s41418-017-0012-4.

46. Wang, P.; Zhen, H.; Jiang, X.; Zhang, W.; Cheng, X.; Guo, G.; Mao, X.; Zhang, X. Boron Neutron Capture Therapy Induces Apoptosis of Glioma Cells through Bcl-2/Bax. BMC Cancer 2010, 10, doi:10.1186/1471-2407-10-661.

47. Matsuya, Y.; Fukunaga, H.; Omura, M.; Date, H. A Model for Estimating Dose-Rate Effects on Cell-Killing of Human Melanoma after Boron Neutron Capture Therapy. Cells 2020, 9, doi:10.3390/cells9051117.

48. Matsuya, Y.; Fukunaga, H.; Omura, M.; Date, H. A Model for Estimating Dose-Rate Effects on Cell-Killing of Human Melanoma after Boron Neutron Capture Therapy. Cells 2020, 9 , doi:10.3390/cells9051117.

49. SEKI, K.; KINASHI, Y.; TAKAHASHI, S. Influence of P53 Status on the Effects of Boron Neutron Capture Therapy in Glioblastoma. Anticancer Research 2015, 35, 169.

50. Rodriguez, C.; Carpano, M.; Curotto, P.; Thorp, S.; Casal, M.; Juvenal, G.; Pisarev, M.; Dagrosa, M.A. In Vitro Studies of DNA Damage and Repair Mechanisms Induced by BNCT in a Poorly Differentiated Thyroid Carcinoma Cell Line. Radiation and Environmental Biophysics 2018, 57, doi:10.1007/s00411-017-0729-y.

51. Nakase, I.; Katayama, M.; Hattori, Y.; Ishimura, M.; Inaura, S.; Fujiwara, D.; TakataniNakase, T.; Fujii, I.; Futaki, S.; Kirihata, M. Intracellular Target Delivery of Cell-Penetrating Peptide-Conjugated Dodecaborate for Boron Neutron Capture Therapy (BNCT). Chemical Communications 2019, 55, doi:10.1039/C9CC03924D.

52. Sun, T.; Zhang, Z.; Li, B.; Chen, G.; Xie, X.; Wei, Y.; Wu, J.; Zhou, Y.; Du, Z. Boron Neutron Capture Therapy Induces Cell Cycle Arrest and Cell Apoptosis of Glioma Stem/Progenitor Cells in Vitro. Radiation Oncology 2013, 8, doi:10.1186/1748-717X-8-195.

53. Wang, P.; Zhen, H.; Jiang, X.; Zhang, W.; Cheng, X.; Guo, G.; Mao, X.; Zhang, X. Boron Neutron Capture Therapy Induces Apoptosis of Glioma Cells through Bcl-2/Bax. BMC Cancer 2010, 10, doi:10.1186/1471-2407-10-661.

54. Sato, T.; Masunaga, S.; Kumada, H.; Hamada, N. Microdosimetric Modeling of Biological Effectiveness for Boron Neutron Capture Therapy Considering Intra- and Intercellular Heterogeneity in 10B Distribution. Scientific Reports 2018, 8, doi:10.1038/s41598-017-188710 .

55. Pedrosa-Rivera, M.; Praena, J.; Porras, I.; Sabariego, M.P.; Köster, U.; Haertlein, M.; Forsyth, V.T.; Ramírez, J.C.; Jover, C.; Jimena, D.; et al. Thermal Neutron Relative Biological Effectiveness Factors for Boron Neutron Capture Therapy from In Vitro Irradiations. Cells 2020, 9, doi:10.3390/cells9102144.

56. Staaf, E.; Brehwens, K.; Haghdoost, S.; Czub, J.; Wojcik, A. Gamma-H2AX Foci in Cells Exposed to a Mixed Beam of X-Rays and Alpha Particles. Genome Integrity 2012, 3, doi:10.1186/2041-9414-3-8.

57. Masutani, M.; Baiseitov, D.; Itoh, T.; Hirai, T.; Berikkhanova, K.; Murakami, Y.; Zhumadilov, Z.; Imahori, Y.; Hoshi, M.; Itami, J. Histological and Biochemical Analysis of 
DNA Damage after BNCT in Rat Model. Applied Radiation and Isotopes 2014, 88, doi:10.1016/j.apradiso.2014.03.003.

58. Kuperman, V.Y. Effect of Dose Rate in Hypofractionated Radiotherapy. Physica Medica 2019, 65, doi:10.1016/j.ejmp.2019.07.005.

59. Faião-Flores, F.; Coelho, P.R.P.; Muniz, R.O.R.; Souza, G.S.; Arruda-Neto, J.; Maria, D.A. Antitumor Potential Induction and Free Radicals Production in Melanoma Cells by Boron Neutron Capture Therapy. Applied Radiation and Isotopes 2011, 69, doi:10.1016/j.apradiso.2011.05.017.

60. Mares, V.; Krajcí, D.; Lisá, V. Subcellular Targets of Mercaptoborate (BSH), a Carrier of 10B for Neutron Capture Therapy (BNCT) of Brain Tumors. Physiological research 2003, 52.

61. Coderre, J.A.; Chanana, A.D.; Joel, D.D.; Elowitz, E.H.; Micca, P.L.; Nawrocky, M.M.; Chadha, M.; Gebbers, J.O.; Shady, M.; Peress, N.S.; et al. Biodistribution of Boronophenylalanine in Patients with Glioblastoma Multiforme: Boron Concentration Correlates with Tumor Cellularity. Radiation Research 1998, 149, doi:10.2307/3579926.

62. Romanov, V.; Isohashi, K.; Alobthani, G.; Beshr, R.; Horitsugi, G.; Kanai, Y.; Naka, S.; Watabe, T.; Shimosegawa, E.; Hatazawa, J. Evaluation of the Total Distribution Volume of 18F-FBPA in Normal Tissues of Healthy Volunteers by Non-Compartmental Kinetic Modeling. Annals of Nuclear Medicine 2020, 34, doi:10.1007/s12149-019-01427-9.

63. Aldossari, S.; McMahon, G.; Lockyer, N.P.; Moore, K.L. Microdistribution and Quantification of the Boron Neutron Capture Therapy Drug BPA in Primary Cell Cultures of Human Glioblastoma Tumour by NanoSIMS. The Analyst 2019, 144, doi:10.1039/C9AN01336A.

64. Fukumitsu, N.; Matsumoto, Y. Development of an Imaging Technique for Boron Neutron Capture Therapy. Cells 2021, 10, doi:10.3390/cells10082135.

65. Menichetti, L.; Cionini, L.; Sauerwein, W.A.; Altieri, S.; Solin, O.; Minn, H.; Salvadori, P.A. Positron Emission Tomography and [18F]BPA: A Perspective Application to Assess Tumour Extraction of Boron in BNCT. Applied Radiation and Isotopes 2009, 67, doi:10.1016/j.apradiso.2009.03.062.

66. Menichetti, L.; Petroni, D.; Panetta, D.; Burchielli, S.; Bortolussi, S.; Matteucci, M.; Pascali, G.; del Turco, S.; del Guerra, A.; Altieri, S.; et al. A Micro-PET/CT Approach Using O-(2[18F]Fluoroethyl)-1-Tyrosine in an Experimental Animal Model of F98 Glioma for BNCT. Applied Radiation and Isotopes 2011, 69, doi:10.1016/j.apradiso.2011.02.037.

67. Shimosegawa, E.; Isohashi, K.; Naka, S.; Horitsugi, G.; Hatazawa, J. Assessment of 10B Concentration in Boron Neutron Capture Therapy: Potential of Image-Guided Therapy Using 18FBPA PET. Annals of Nuclear Medicine 2016, 30, doi:10.1007/s12149-016-1121-8.

68. Li, J.; Shi, Y.; Zhang, Z.; Liu, H.; Lang, L.; Liu, T.; Chen, X.; Liu, Z. A Metabolically Stable Boron-Derived Tyrosine Serves as a Theranostic Agent for Positron Emission Tomography Guided Boron Neutron Capture Therapy. Bioconjugate Chemistry 2019, 30, doi:10.1021/acs.bioconjchem.9b00578.

69. Alberti, D.; Deagostino, A.; Toppino, A.; Protti, N.; Bortolussi, S.; Altieri, S.; Aime, S.; Geninatti Crich, S. An Innovative Therapeutic Approach for Malignant Mesothelioma 
Treatment Based on the Use of Gd/Boron Multimodal Probes for MRI Guided BNCT. Journal of Controlled Release 2018, 280, 31-38, doi:10.1016/j.jconrel.2018.04.043.

70. Takahashi, K.; Nakamura, H.; Furumoto, S.; Yamamoto, K.; Fukuda, H.; Matsumura, A.; Yamamoto, Y. Synthesis and in Vivo Biodistribution of BPA-Gd-DTPA Complex as a Potential MRI Contrast Carrier for Neutron Capture Therapy. Bioorganic \& Medicinal Chemistry 2005, 13, doi:10.1016/j.bmc.2004.10.046.

71. Bonora, M.; Corti, M.; Borsa, F.; Bortolussi, S.; Protti, N.; Santoro, D.; Stella, S.; Altieri, S.; Zonta, C.; Clerici, A.M.; et al. 1H and 10B NMR and MRI Investigation of Boron- and Gadolinium-Boron Compounds in Boron Neutron Capture Therapy. Applied Radiation and Isotopes 2011, 69, doi:10.1016/j.apradiso.2011.02.021.

72. Geninatti-Crich, S.; Deagostino, A.; Toppino, A.; Alberti, D.; Venturello, P.; Aime, S. Boronated Compounds for Imaging Guided BNCT Applications. Anti-Cancer Agents in Medicinal Chemistry 2012, 12, doi:10.2174/187152012800617786.

73. Balcerzyk, M.; De-Miguel, M.; Guerrero, C.; Fernandez, B. Quantification of Boron Compound Concentration for BNCT Using Positron Emission Tomography. Cells 2020, 9, doi:10.3390/cells9092084.

74. CHANDRA, S.; AHMAD, T.; BARTH, R.F.; KABALKA, G.W. Quantitative Evaluation of Boron Neutron Capture Therapy (BNCT) Drugs for Boron Delivery and Retention at Subcellular-Scale Resolution in Human Glioblastoma Cells with Imaging Secondary Ion Mass Spectrometry (SIMS). Journal of Microscopy 2014, 254, doi:10.1111/jmi.12126.

75. Lanni, E.J.; Rubakhin, S.S.; Sweedler, J. v. Mass Spectrometry Imaging and Profiling of Single Cells. Journal of Proteomics 2012, 75, doi:10.1016/j.jprot.2012.03.017.

76. Barth, R.F.; Kabalka, G.W.; Yang, W.; Huo, T.; Nakkula, R.J.; Shaikh, A.L.; Haider, S.A.; Chandra, S. Evaluation of Unnatural Cyclic Amino Acids as Boron Delivery Agents for Treatment of Melanomas and Gliomas. Applied Radiation and Isotopes 2014, 88, doi:10.1016/j.apradiso.2013.11.133.

77. Chandra, S.; Barth, R.F.; Haider, S.A.; Yang, W.; Huo, T.; Shaikh, A.L.; Kabalka, G.W. Biodistribution and Subcellular Localization of an Unnatural Boron-Containing Amino Acid (Cis-ABCPC) by Imaging Secondary Ion Mass Spectrometry for Neutron Capture Therapy of Melanomas and Gliomas. PLOS ONE 2013, 8, doi:10.1371/journal.pone.0075377.

78. CHANDRA, S.; AHMAD, T.; BARTH, R.F.; KABALKA, G.W. Quantitative Evaluation of Boron Neutron Capture Therapy (BNCT) Drugs for Boron Delivery and Retention at Subcellular-Scale Resolution in Human Glioblastoma Cells with Imaging Secondary Ion Mass Spectrometry (SIMS). Journal of Microscopy 2014, 254, doi:10.1111/jmi.12126.

79. Aldossari, S.; McMahon, G.; Lockyer, N.P.; Moore, K.L. Microdistribution and Quantification of the Boron Neutron Capture Therapy Drug BPA in Primary Cell Cultures of Human Glioblastoma Tumour by NanoSIMS. The Analyst 2019, 144, doi:10.1039/C9AN01336A.

80. Minsky, D.M.; Valda, A.A.; Kreiner, A.J.; Green, S.; Wojnecki, C.; Ghani, Z. First Tomographic Image of Neutron Capture Rate in a BNCT Facility. Applied Radiation and Isotopes 2011, 69, doi:10.1016/j.apradiso.2011.01.030. 
81. Minsky, D.M.; Valda, A.A.; Kreiner, A.J.; Green, S.; Wojnecki, C.; Ghani, Z. Experimental Feasibility Studies on a SPECT Tomograph for BNCT Dosimetry. Applied Radiation and Isotopes 2009, 67, doi:10.1016/j.apradiso.2009.03.044.

82. Winkler, A.; Koivunoro, H.; Reijonen, V.; Auterinen, I.; Savolainen, S. Prompt Gamma and Neutron Detection in BNCT Utilizing a CdTe Detector. Applied Radiation and Isotopes 2015, 106, doi:10.1016/j.apradiso.2015.07.040.

83. Fatemi, S.; Altieri, S.; Bortolussi, S.; Postuma, I.; Benassi, G.; Zambelli, N.; Bettelli, M.; Zanichelli, M.; Zappettini, A.; Protti, N. Preliminary Characterization of a CdZnTe Photon Detector for BNCT-SPECT. Nuclear Instruments and Methods in Physics Research Section A: Accelerators, Spectrometers, Detectors and Associated Equipment 2018, 903, doi:10.1016/j.nima.2018.06.068.

84. Fatemi, S.; Gong, C.H.; Bortolussi, S.; Magni, C.; Postuma, I.; Bettelli, M.; Benassi, G.; Zambelli, N.; Zappettini, A.; Tang, X.B.; et al. Innovative 3D Sensitive CdZnTe Solid State Detector for Dose Monitoring in Boron Neutron Capture Therapy (BNCT). Nuclear Instruments and Methods in Physics Research Section A: Accelerators, Spectrometers, Detectors and Associated Equipment 2019, 936, doi:10.1016/j.nima.2018.09.135.

85. Murata, I.; Mukai, T.; Nakamura, S.; Miyamaru, H.; Kato, I. Development of a Thick CdTe Detector for BNCT-SPECT. Applied Radiation and Isotopes 2011, 69, doi:10.1016/j.apradiso.2011.05.014.

86. Murata, I.; Nakamura, S.; Manabe, M.; Miyamaru, H.; Kato, I. Characterization Measurement of a Thick CdTe Detector for BNCT-SPECT - Detection Efficiency and Energy Resolution. Applied Radiation and Isotopes 2014, 88, doi:10.1016/j.apradiso.2014.01.023.

87. Kim, M.; Hong, B.H.; Cho, I.; Park, C.; Min, S.-H.; Hwang, W.T.; Lee, W.; Kim, K.M. Design of a Scintillator-Based Prompt Gamma Camera for Boron-Neutron Capture Therapy: Comparison of SrI2 and GAGG Using Monte-Carlo Simulation. Nuclear Engineering and Technology 2021, 53, doi:10.1016/j.net.2020.07.010.

88. Hu, N.; Tanaka, H.; Takata, T.; Endo, S.; Masunaga, S.; Suzuki, M.; Sakurai, Y. Evaluation of PHITS for Microdosimetry in BNCT to Support Radiobiological Research. Applied Radiation and Isotopes 2020, 161, doi:10.1016/j.apradiso.2020.109148.

89. Sato, T.; Masunaga, S.; Kumada, H.; Hamada, N. Microdosimetric Modeling of Biological Effectiveness for Boron Neutron Capture Therapy Considering Intra- and Intercellular Heterogeneity in 10B Distribution. Scientific Reports 2018, 8, doi:10.1038/s41598-017-188710 . 